\title{
Multi-dimensional explorations in supernova theory
}

\author{
Adam Burrows $^{\mathrm{a}, *}$, Luc Dessart $^{\mathrm{a}}$, Christian D. Ott $^{\mathrm{a}, \mathrm{b}}$, Eli Livne ${ }^{\mathrm{c}}$ \\ ${ }^{a}$ Department of Astronomy, The University of Arizona, Tucson, AZ 85721, USA \\ ${ }^{\mathrm{b}}$ Albert-Einstein-Institut, Max-Planck-Institut für Gravitationsphysik, Potsdam, Germany \\ ${ }^{\mathrm{c}}$ Racah Institute of Physics, The Hebrew University, Jerusalem, Israel \\ Available online 17 February 2007 \\ editor: G.E. Brown
}

\begin{abstract}
In this paper, we bring together various of our published and unpublished findings from our recent 2D multi-group, flux-limited radiation hydrodynamic simulations of the collapse and explosion of the cores of massive stars. Aided by 2D and 3D graphical renditions, we motivate the acoustic mechanism of core-collapse supernova explosions and explain, as best we currently can, the phases and phenomena that attend this mechanism. Two major foci of our presentation are the outer shock instability and the inner core g-mode oscillations. The former sets the stage for the latter, which damp by the generation of sound. This sound propagates outward to energize the explosion and is relevant only if the core has not exploded earlier by some other means. Hence, it is a more delayed mechanism than the traditional neutrino mechanism that has been studied for the last twenty years since it was championed by Bethe and Wilson. We discuss protoneutron star convection, accretion-induced-collapse, gravitational wave emissions, pulsar kicks, the angular anisotropy of the neutrino emissions, a subset of numerical issues, and a new code we are designing that should supercede our current supernova code VULCAN/2D. Whatever ideas last from this current generation of numerical results, and whatever the eventual mechanism(s), we conclude that the breaking of spherical symmetry will survive as one of the crucial keys to the supernova puzzle.
\end{abstract}

(C) 2007 Elsevier B.V. All rights reserved.

PACS: 97.30.-b

Keywords: Supernova theory; Multi-dimensional simulations; Neutrinos; Acoustic power; Stellar pulsations

\section{Contents}

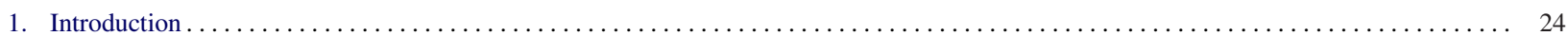

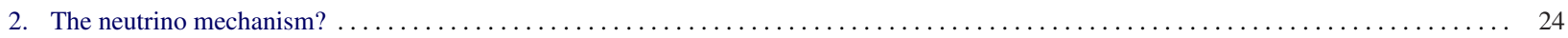

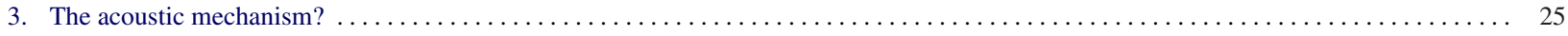

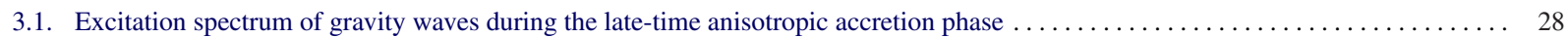

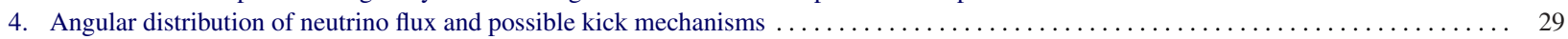

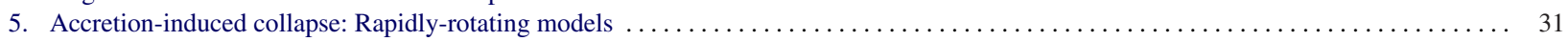

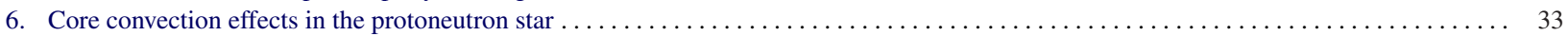

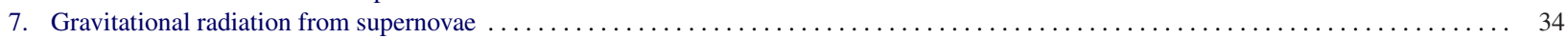

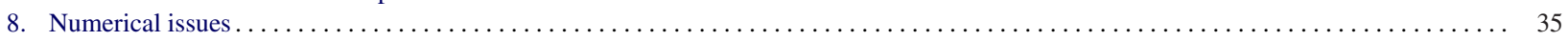

\footnotetext{
* Corresponding author.

E-mail address: aburrows@ as.arizona.edu (A. Burrows).
} 
9. BETHE: a new multi-dimensional radiation hydrodynamic code $\ldots \ldots \ldots \ldots \ldots$

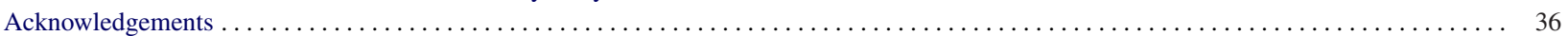

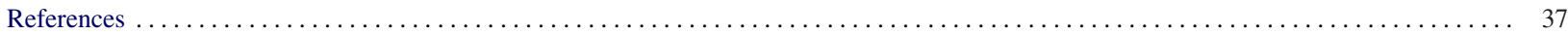

\section{Introduction}

Over the past 45 years, the theory of core-collapse supernova explosions and attendant phenomena has witnessed evolution, elaboration, clarification, and much speculation. Many ideas have come and gone, but in the process the physics, initial conditions, and numerical sophistication have all greatly improved. Such has been the progress that there are some who believe that the physical inputs are in hand and it is only the numerical tools that need sharpening and development. That may be, but even if this were the case such is the complexity of the three-dimensional radiation hydrodynamics (magneto-hydrodynamics?) which Nature integrates effortlessly and in real time that astrophysicists are still debating the primary agents of explosion. The major drivers that remain are neutrinos, acoustic power, and magnetic stresses. The neutrino heating mechanism is still the favorite, but both acoustic power due to the excitation of inner core pulsations and magnetic hoop stresses generated in a rapidly differentially rotating protoneutron star core are contenders. It may be a mix of all three.

The central issues of supernova explosion theory are: (1) the mechanism of explosion; (2) the nucleosynthetic yields, including ${ }^{56} \mathrm{Ni}$, the rp-process, and the r-process; (3) the blast morphology (and the associated polarization of the emergent light); (4) the origin of pulsar kicks; (5) the origin of pulsar spins; (6) the generation and distribution of pulsar, AXP, and magnetar B-fields; (7) black hole formation; (8) the systematics of all these things with progenitor, its mass, metallicity, and rotational profile; and (9) the connection with gamma-ray bursts. We are still a long way from achieving this program, but there are new ideas and numerical results that are beginning to resemble, however imperfectly, a suggestive idealization of Nature. In this paper, we review and synthesize our emerging perspectives on some of these issues, with a bias towards our own work and thoughts. To do so, we have bought together in one place various conclusions that can be found scattered in many of our recently published works, as well as new results and graphics that add to the overall analysis and discussion. What we conclude is that, whatever the central mechanism of explosion, the breaking of spherical symmetry, multi-dimensional effects, and instabilities are keys to the supernova puzzle. This is the theme, if there is one, of this monograph. Not only do pulsar kicks hint at this, but all of the most sophisticated simulations recently undertaken point in this direction, whether the mechanism is via neutrinos or sound. What survives of this current generation of simulation results and ideas will depend upon the next generation of calculations, and the addition of adequate 3D simulations and analysis. Hence, we provide here but a snapshot of the moving theoretical terrain.

\section{The neutrino mechanism?}

The neutrino-heating mechanism, in which a stalled bounce shock is reenergized by neutrino energy deposition after a slight delay, perhaps aided by overturning instabilities in this "gain region," has been the working hypothesis of supernova theorists for the last 20 years [1-4]. Past calculations in support of this mechanism, or variations on its theme, include those by Wilson and Mayle [5,6], Mayle and Wilson [7], Herant et al. [8], Burrows et al. [9], Janka and Müller [10], and Fryer and Warren [11,12]. Convection was thought to facilitate explosion by increasing the dwell time in the gain region of parcels of matter accreting through the shock, and thereby the efficiency of neutrino heating. In $1 \mathrm{D}$, such parcels move quickly through the gain region, achieve the cooling region, and settle upon the core before the explosion condition is met. Fig. 1 depicts examples of the circuitous routes taken by accreting elements after they penetrate through the shock. However, recent calculations employing careful neutrino physics and numerics suggest that the neutrino mechanism, when it succeeds, may at best be marginal. Kitaura et al. [13] follow in spherical symmetry the compact 1.38- $\mathrm{M}_{\odot} \mathrm{O}-\mathrm{Ne}-\mathrm{Mg}$ core of the $8.8-\mathrm{M}_{\odot}$ model of Nomoto and Hashimoto [14], with a very tenuous outer envelope, and obtain a delayed neutrino-driven explosion. However, the explosion energy is only $\gtrsim 10^{50} \mathrm{ergs}$. For the slightly more massive 11.2-M $\odot$ progenitor of Woosley et al. [15] (WHW02), Buras et al. [3] witness the onset, not in 1D, but in 2D, of a neutrino-driven dipolar explosion aided by the advective-acoustic standing-accretion-shock-instability (SASI) $[2,3,16-24]$. They also infer a very weak explosion energy, this time near $10^{49} \mathrm{ergs}$, not correcting for neutrino 

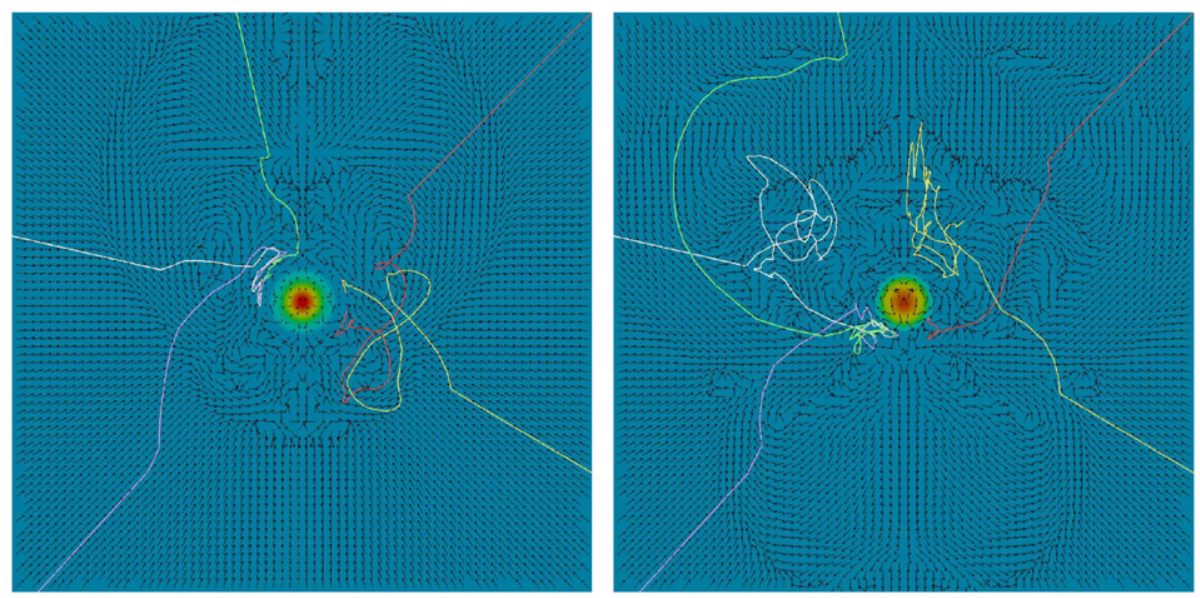

Fig. 1. Two renderings of five representative streak lines showing the paths of infalling parcels of matter during a period of $\sim 100 \mathrm{~ms}$. The starting points were two different random times during the SASI phase and before explosion. Clearly seen is the encounter with the shock and the subsequent effects of the turbulent/convective post-shock environment. The velocity vector fields in the backgrounds are for the "final" times. In 1D, these would be straight lines along a radius. In this representative $2 \mathrm{D}$ simulation, the matter is shown to dwell a bit longer in the outer shocked region before it settles onto the inner core.

driving subsequent to the early termination of their calculation or for the binding energy of the outer envelope. Buras et al. [3] focus on the importance of calculating over the full $180^{\circ} 2 \mathrm{D}$ domain so as not to suppress the $\ell=1$ mode of the SASI, since they do not obtain even a weak explosion when constraining the computational domain to $90^{\circ}$. Importantly, the breaking of spherical symmetry is a key to the explosion they see, an emerging theme that we endorse for the majority of core-collapse explosions. However, Buras et al. [3] do not as yet see any indications that more massive progenitors explode by the neutrino mechanism, even when aided by the SASI and convection.

\section{The acoustic mechanism?}

If the neutrino mechanism is eventually shown to work in the first few hundred milliseconds after bounce, perhaps due to $3 \mathrm{D}$ effects or better physics and numerics, this will be welcome news to supernova theorists. However, what if most of the time it does not? Recently, Burrows et al. [22,23], using the code VULCAN/2D, have suggested an explosion driver that relies predominantly on acoustic power generated by vigorous core pulsations. First, within $\sim 200 \mathrm{~ms}$ after bounce the stalled shock experiences the SASI with a period of $\sim 15-30 \mathrm{~ms}$ and begins to execute large deviations from sphericity. The growth of this outer shock instability saturates due to the generation of secondary shocks. Our calculations support the notion that all non-rotating progenitors that do not explode by an early neutrino mechanism experience the SASI, whose oscillation periods are best interpreted simply as the sound-travel-times across the shocked regions. However, this instability is not the primary agent of explosion. Rather, it is the acoustic power generated early on in the inner turbulent region stirred by the SASI-generated accretion plumes, and most importantly, but later on, by the consequent excitation and sonic damping of rapid core g-mode pulsations. An $\ell=1 \mathrm{~g}$-mode (not the SASI) with a period of $\sim 3 \mathrm{~ms}$ grows at late times to be prominent around $\sim 500 \mathrm{~ms}$ after bounce. Fig. 2 portrays the temporal evolution of core oscillation frequencies for a representative model calculation. Fig. 3 provides a 3D snapshot in isodensity sheets of the $\ell=1$ dipolar pulsation of the inner core in a calculation from Burrows et al. [22,23]. The accreting protoneutron star (PNS) then acts like a self-excited oscillator (see Section 3.1). The sound pulses radiated from the core steepen into shock waves that merge as they propagate into the outer mantle and deposit their energy and momentum with high efficiency. The ultimate source of the acoustic power is the gravitational energy of infall. We find that the associated acoustic power is sufficient to drive the explosion of all models studied to date $>500-1100 \mathrm{~ms}$ after bounce. The delay to explosion and its sudden onset is depicted in Fig. 4. The colors represent the Mach number of the matter. Note that the Mach number of the turbulence behind the shock increase with time, and that the secondary shock waves that emerge during the non-linear phase of the SASI and during the explosion are clearly in evidence. The angular distribution of the emitted sound is fundamentally aspherical and the acoustic power fills a growing cavity 


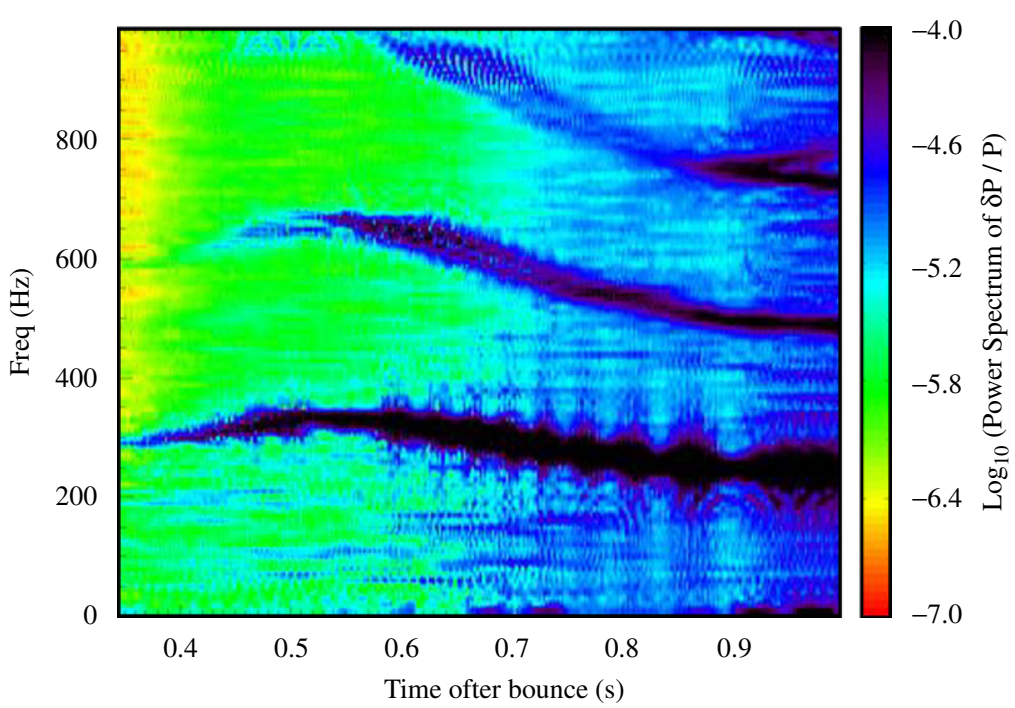

Fig. 2. Colormap of the power spectrum of the fractional pressure variation $\left(P(R, \theta)-\langle P(R, \theta)\rangle_{\theta}\right) /\langle P(R, \theta)\rangle_{\theta}$ at a radius $R=30 \mathrm{~km}$, as a function of time after bounce and frequency. For each time, a power spectrum is calculated from a sample of time snapshots covering $t \pm 50 \mathrm{~ms}$, at a resolution of $0.5 \mathrm{~ms}$. Note the emergence of power in the $\sim 330 \mathrm{~Hz}(\equiv 3 \mathrm{~ms}) \ell=1 \mathrm{~g}$-mode, as well as the strengthening at late times of the first harmonic oscillation near $\sim 675 \mathrm{~Hz}$. The $\ell=2$ component of the latter is of relevance for gravitational radiation emission. Note also the late-time evolution of the characteristic frequencies. Figure taken from the study by Burrows et al. [22].

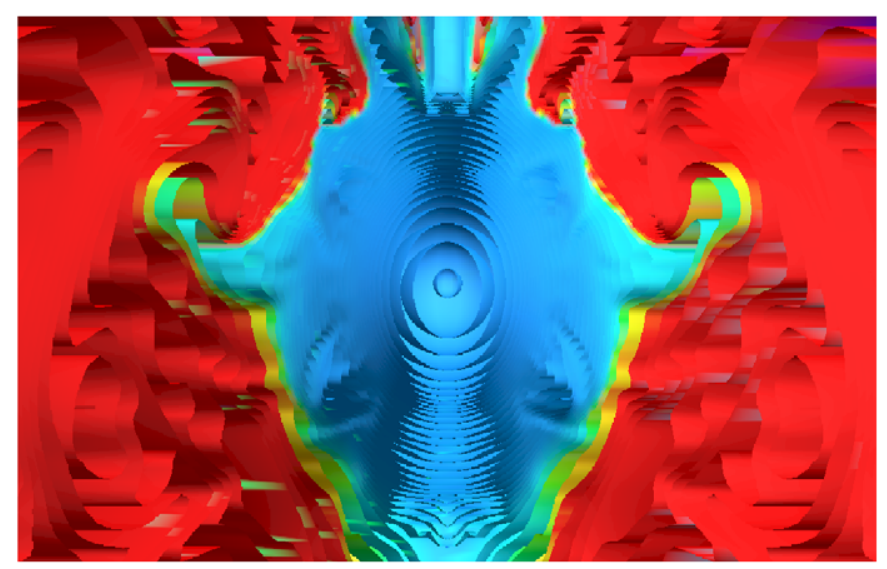

Fig. 3. 3D plot depicting isodensity sheets in the core of the protoneutron star (PNS) at late times after the core oscillation has achieved significant amplitudes. The linear scale is about $50 \mathrm{~km}$. The inner blue region is at low entropies and the outer red region is turbulent and at much higher entropies. The relative displacement of the two inner shells due to the $\ell=1 \mathrm{~g}$-mode is discernible.

that drives the explosion. The evolution of such a cavity for a representative core-acoustic explosion is presented in Fig. 5. An advantage of the acoustic mechanism is that acoustic power does not abate until accretion subsides, so that it is available as long as it may be needed to explode the star.

There are certain other virtues to acoustic driving that bear mentioning. First is that while the acoustic luminosity is much smaller than the neutrino luminosity, almost all of the sound is absorbed in the mantle matter. At late times in our simulation, less than a percent of the $v_{e}$ and $\bar{v}_{e}$ neutrino luminosity is absorbed. This amounts to an neutrino absorption power of $\leqslant 10^{50} \mathrm{erg} \mathrm{s}^{-1}$, compared with an estimated core acoustic power at the end of our calculations near $\sim 10^{51} \mathrm{erg} \mathrm{s}^{-1}$. Second, sound carries not only energy, but momentum, and this factor seems to be important in our simulations. The momentum flux for sound with the same energy flux as neutrino radiation is larger by the ratio of 


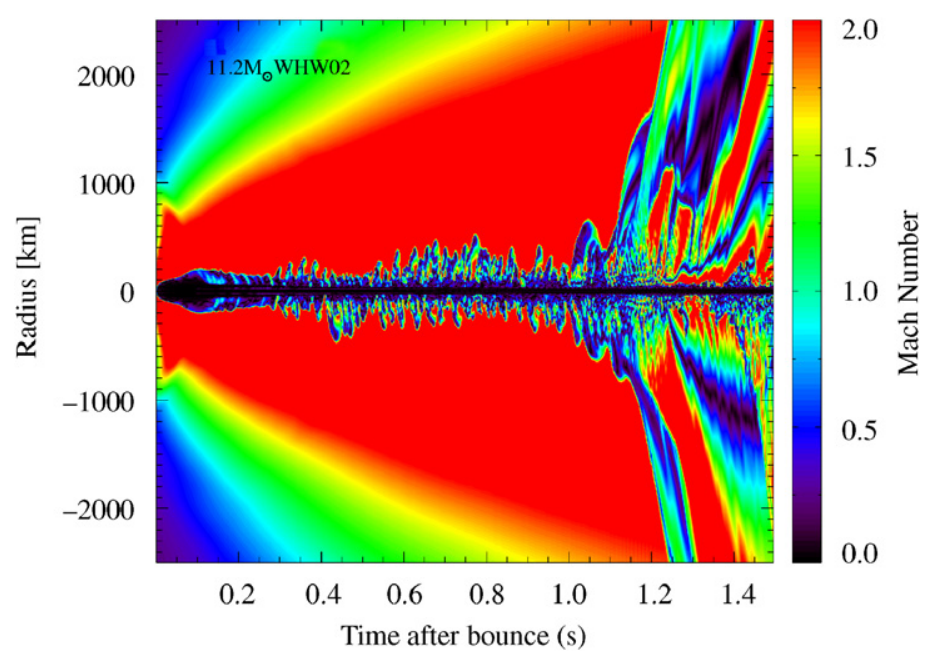

Fig. 4. Colormap of the time evolution after bounce of the Mach number profile along the poles (in both the positive and the negative directions) for the 11.2-M $\odot$ model of WHW02. The Mach number is saturated at a value of two to enhance the contrast. The large red region is infalling material. The position of the bounce shock (near $\pm 200-500 \mathrm{~km}$ ) and its oscillations before explosion due to the SASI are clearly seen, as are the multiple shocks that emerge asymmetrically at late times to power the explosion.
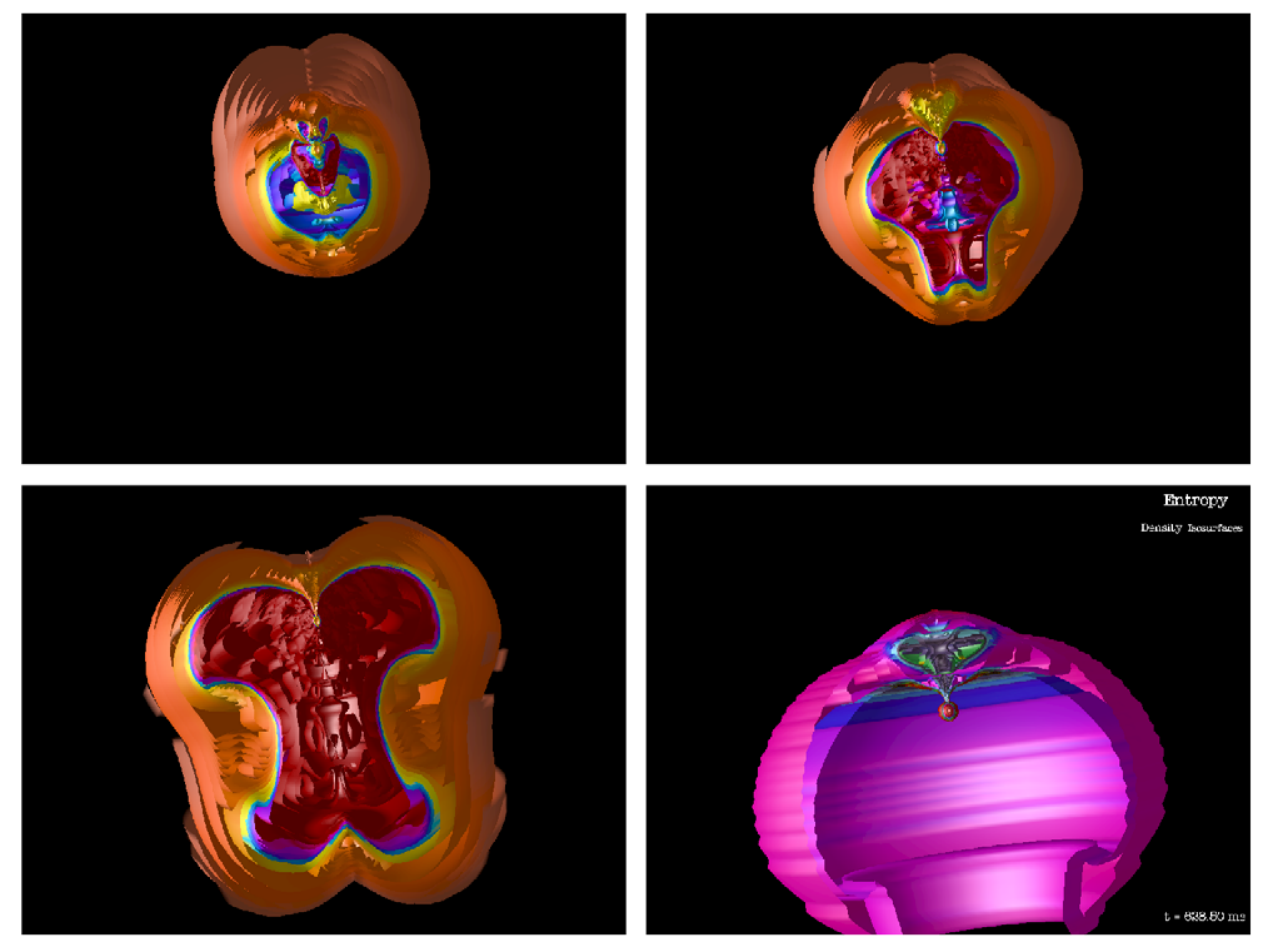

Fig. 5. A temporal sequence of 3D renditions of isodensity sheets painted with entropy values for a 2D model exploding by the anisotropic acoustic mechanism. The explosion is predominantly along one axis, during which accretion streams (here near the top) continue to impinge upon the core. There is simultaneous accretion and explosion. The central sphere is the protoneutron star and an expanding cavity is progressively excavated and filled with acoustic power and thermal energy. The scale for the first three plots is approximately $5000 \mathrm{~km}$ on a side, while that for the last one is approximately $10000 \mathrm{~km}$.

the speed of light to the speed of sound, which in the inner mantle regions is as much as a factor of ten. Third, acoustic power propagates from where it is generated to where it is needed; it fulfills the central requirement of a core-collapse supernova mechanism that it involve energy transfer from the bound interior PNS to the outer exploding mantle. 
If the acoustic power is large enough, it is the ideal transfer agent. Fourth, the acoustic source seems to grow just when the neutrino luminosity is ebbing and, importantly, it continues until explosion ensues. Fifth, the successive merger of trains of sound waves that steepen into shocks provides a non-neutrino way to entropize some of the matter and naturally achieve r-process conditions.

It should be pointed out that the SASI and the breaking of spherical symmetry are likely important even in the context of the neutrino-driven explosion scenario, and in fact in every viable explosion scenario that is emerging from modern calculations. When the SASI is in its vigorous non-linear phase, its $\ell=1$ oscillations result in quasi-periodic fluctuations in the effective accretion rate and ram pressure on any given side of the inner core. In the canonical neutrino-driven mechanism of supernova explosions, when and after the explosion occurs the pressure around the neutrinospheres decays. When this pressure is sufficiently low, a neutrino-driven wind spontaneously emerges from the inner core, announced and preceded by a secondary shock wave [25,9]. This is what happens in the standard neutrino-driven scenario when the flow is semi-spherical. However, the SASI can set up a situation in which the pressure and ram pressure on one side render that side of the core unstable to the emergence of a neutrino-driven wind even before the canonical explosion. In fact, this wind can be the explosion itself and need not be preceded by a primary explosion. This is what Buras et al. [3] see for their 11.2- $\mathrm{M}_{\odot}$ simulation. However, such an explosion seems generically underenergetic. In the acoustic mechanism, the neutrinos are replaced/dominated by the acoustic power, but the general paradigm in which the SASI leads temporarily/periodically to lower pressures on one side of the core that enable the emergence of an asymmetric wind still obtains. In any case, an aspherical "wind" is a good description of the supernova explosion [23,25-27] and the breaking of spherical symmetry is the key. The latter can also enable simultaneous accretion and explosion, thereby solving the problem of the accretion tamp that has bedeviled the theory of the neutrino mechanism for years.

Hence, we see in the breaking of spherical symmetry in our simulations and in the often unipolar nature of the resulting explosions a natural explanation for the polarizations observed in the inner debris of Type Ic [28] and Type II [29] supernovae. Inner asymmetries of 2:1 or 3:1 are easily obtained in this model and do not require MHD jets. Fig. 6 compares explosion morphologies for a representative range of VULCAN/2D simulations. The actual degree and character of the anisotropy is unpredictable, given the chaotic dynamics, but spherical explosions should be exceedingly rare.

\subsection{Excitation spectrum of gravity waves during the late-time anisotropic accretion phase}

The explosions we see resemble strong anisotropic winds. A spherical wind imparts no net momentum to the residue; an asymmetric wind imparts a kick and "ablation" force on the accretion streams and core. The recoil implied is a purely hydrodynamical mechanism, whatever the agency of explosion (be it neutrinos or sound), and has two results. First, the recoil due to the anisotropic wind pushes the accretion streams to the opposite side, making the accretion very anisotropic. A fraction of the gravitational energy of accretion is used to continue to excite the inner core gmode oscillation. Because the accretion funnels are supersonic, the coupling to the core is non-linear. Importantly, the oscillation of the core cannot do work back on the exciting accretion stream(s) that would otherwise damp the core oscillation; any work done is accreted back. Hence, the analogy with the swing which requires a resonance or near resonance to achieve significant amplitude is not germane. A steady stream onto the core can continue to power the periodic core oscillation, even though there is no intrinsic periodicity to the accretion. The accretion funnel does have a width, which like a rock hitting a pond has associated with it a range of characteristic sizes (read wavelengths). Due to the dispersion relation of gravity waves between wavelength and frequency, a whole period spectrum of ripples is generated which contains the period of the $\ell=1$ core g-mode (as well as those of many of the higher- $\ell$ core g-modes).

For instance, if the steady accretion stream has a width of $\sim 10 \mathrm{~km}$, the average frequency of the gravity waves generated at the collision with the inner core whose surface gravity is $\sim 10^{13} \mathrm{~cm} \mathrm{~s}^{-2}$ is $\sim 700 \mathrm{~Hz}$, with a spectrum that extends from lower to higher frequencies that comfortably overlaps with the core g-mode eigenfrequencies. This is true despite the fact that there is no such periodicity in the exciting accretion stream itself. The thinner the stream the wider the excitation frequency spectrum. This limit is analogous to the "white noise" spectrum of gravity waves excited by the narrow bow of a ship that collectively result in the characteristic scale-free, fixed-angle bow wave. What is not clear from merely analytic arguments is the fraction of the accretion power that should be channeled into mechanical energy. The fraction channeled into neutrino losses is usually the larger. One can estimate in the crudest fashion the power being pumped into gravity waves as a function of the stream radius, $R_{0}$, the wave height, $H_{0}$, the average density 

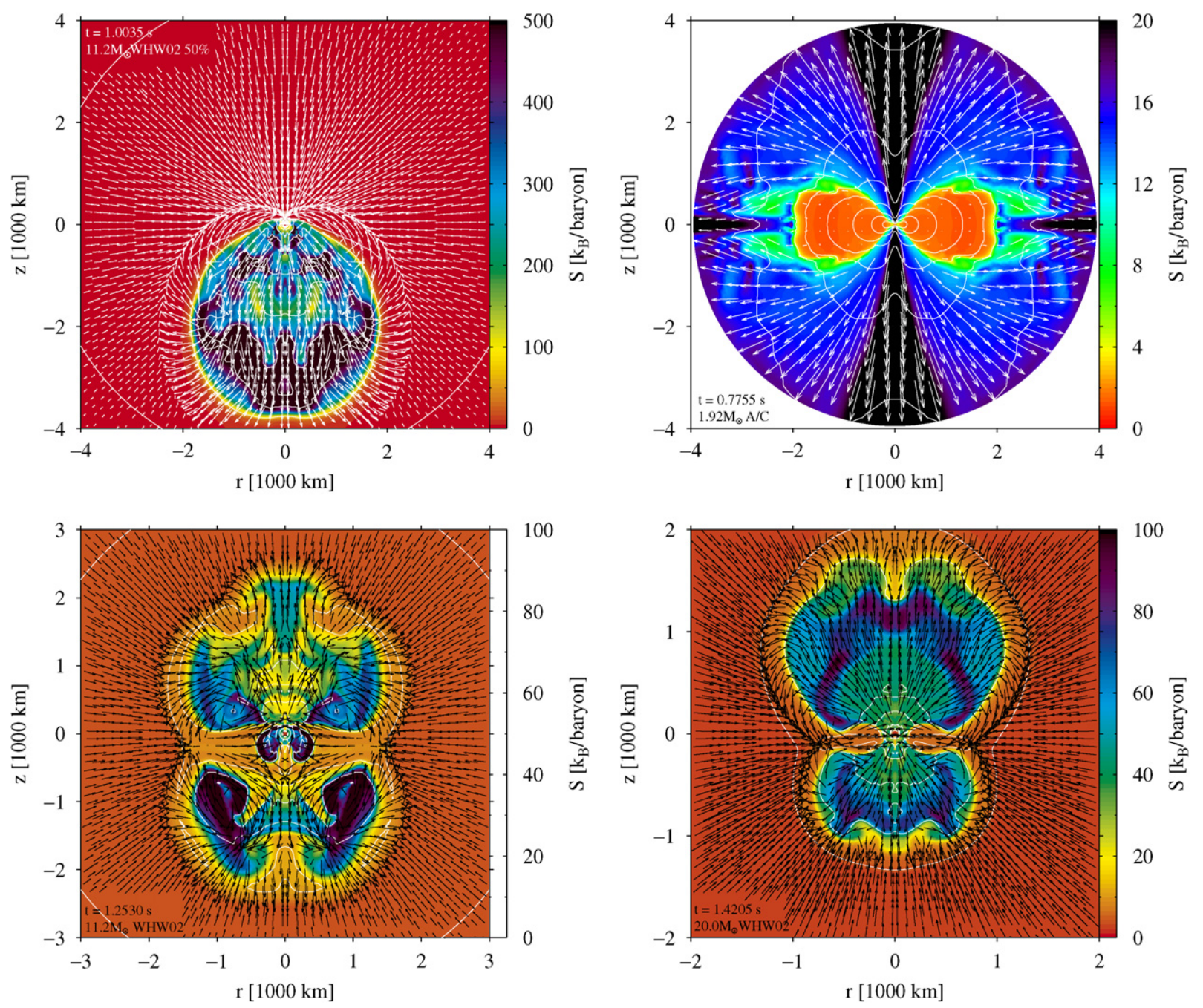

Fig. 6. Colormap of the entropy for our simulation of the 11.2-M $\odot$ model of WHW02, but with the neutral-current scattering rates diminished artificially by $50 \%$ (top left), the $1.92-\mathrm{M}_{\odot}$ AIC model of Dessart et al. [32] (top right), the default 11.2-M $\odot$ model of WHW02 (bottom left), and the 20-M $\odot$ model of WHW02 (bottom right). Times after bounce are indicated in each panel. The maximum length vector, set to $5 \%$ of the width of the display, corresponds to a velocity of $10000 \mathrm{~km} \mathrm{~s}^{-1}$ (values above that are saturated), except for the AIC model for which the corresponding values are $10 \%$ and $20000 \mathrm{~km} \mathrm{~s}^{-1}$.

at the core "surface," $\rho$, and the surface gravity, $g$ :

$$
\dot{E} \sim \frac{\pi \rho}{2}\left(g R_{0}\right)^{3 / 2} H_{0}^{2} \sim 0.5 \times 10^{51} \mathrm{erg} \mathrm{s}^{-1} \rho_{11}\left(g_{13}\right)^{3 / 2}\left(\frac{R_{0}}{10 \mathrm{~km}}\right)^{3 / 2}\left(\frac{H_{0}}{3 \mathrm{~km}}\right)^{2},
$$

where $\rho_{11}=\rho /\left(10^{11} \mathrm{~g} \mathrm{~cm}^{-3}\right)$ and $g_{13}=g /\left(10^{13} \mathrm{~cm} \mathrm{~s}^{-2}\right)$. The reference value of $\dot{E}$ in Eq. (1) is suggestive. To estimate $H_{0}$ we would need the fraction of the accretion power that should be set equal to $\dot{E}$, something currently we can get only from imperfect numerical simulations. However, the reference numbers in Eq. (1) come from such simulations.

\section{Angular distribution of neutrino flux and possible kick mechanisms}

With VULCAN/2D, Burrows et al. [23] are able to ascertain the magnitude and sign of the impulse due to anisotropic neutrino emissions. We find that during our simulations (to approximately $1.5 \mathrm{~s}$ after bounce) the neutrino recoil effect 

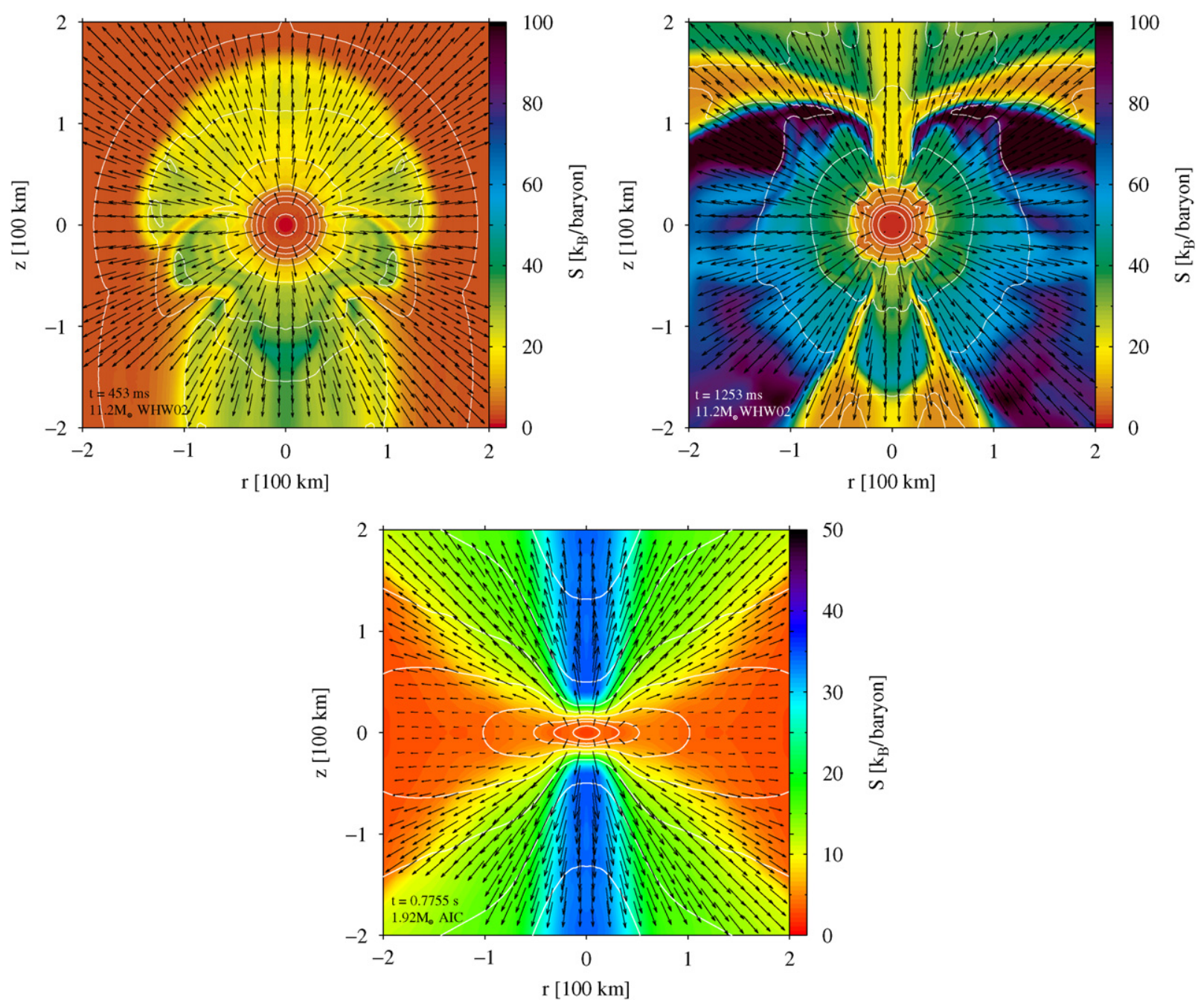

Fig. 7. Colormap of the entropy for the 11.2-M $\odot$ model of WHW02, at 453 (left) and $1253 \mathrm{~ms}$ (center) after bounce [23], and for the 1.92-M $\odot$ accretion-induced collapse (AIC) white dwarf model of Dessart et al. [32] at $775.5 \mathrm{~ms}$ after bounce (bottom). Density contours are also drawn in white, for every decade down from $10^{14} \mathrm{~g} \mathrm{~cm}^{-3}$ (innermost and spherical ring). We overplot vectors of the quantity $4 \pi R^{2} \vec{F}_{v}$ (where $\vec{F}_{v}$ is the total neutrino flux), and saturate this quantity at $1.2 \times 10^{53} \mathrm{erg} \mathrm{s}^{-1}$ with a maximum length of $5 \%$ of the display width. Note how the neutrino flux remains essentially radial in the non-rotating model (left and center), despite the large deviations from spherical symmetry in the entropy and density distributions. By contrast, in the fast rotating model of the AIC of Dessart et al. [32] significant asphericity prevails in the high-density regions which modify considerably the emergent neutrino flux, enhanced along the pole, but diminished along the equator.

on the core is not large, at most $\sim 50 \mathrm{~km} \mathrm{~s}^{-1}$, but that by the end of our simulations it is still growing and is in the opposite direction to the blast. Moreover, after the explosion commences, the impulses on the protoneutron star due to the matter ejecta and the neutrino radiation $a d d$. The small magnitude of the neutrino force during the delay to explosion may seem inconsistent with the very anisotropic accretion. However, the radiation field is much smoother by its nature than the material field. Fig. 7 provides vector fields of instantaneous neutrino flux distributions superposed on background entropy maps for three different models. Only for the accretion-induced-collapse model (see Section 5 ) is the flux very aspherical, and this is due to the very rapid rotation. For the non-rotating models, the neutrinos are not radiated instantaneously upon compression in an accretion column onto the protoneutron star. The matter is too opaque for immediate reradiation. Rather, the neutrinos emerge after the compressed accreta have spread more uniformly over the inner core, and, therefore, are radiated much more isotropically than the matter is accreted. But importantly, after explosion the neutrinos can emerge more easily along the direction of the blast, since the material 

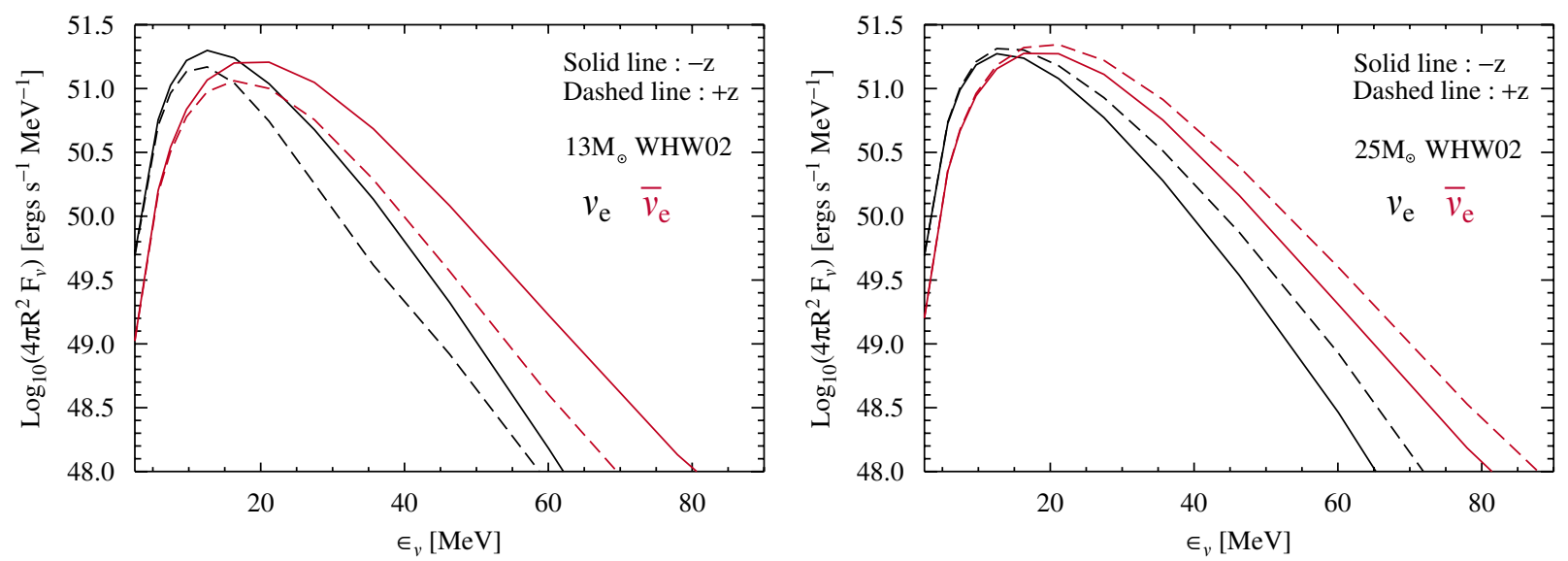

Fig. 8. Left: Energy spectra of the electron-neutrino (black) and anti-electron neutrino (red) fluxes along the poles (solid line: negative $z$-direction; dashed line: positive $z$-direction) for the $13-\mathrm{M}_{\odot}$ model of WHW02. The fluxes are multiplied by a factor $4 \pi R^{2}$ and are at $1.44 \mathrm{~s}$ after bounce and after the explosion commences. Right: Same as at the left, but for the $25-\mathrm{M}_{\odot}$ model of WHW02 at $1.42 \mathrm{~s}$ after bounce. Note that the hotter and higher fluxes are in each case in the direction of the explosion, though the two models shown explode in different directions. Figure taken from Burrows et al. 2006b.

around the neutrinospheres thins out in this direction, and not along the direction experiencing continuing accretion, which, as stated, is more opaque. Fig. 8 depicts the spectra of the $v_{e}$ and $\bar{v}_{e}$ neutrinos in the up and down directions (along the poles) near the end of the simulations of the $13-\mathrm{M}_{\odot}$ and $25-\mathrm{M}_{\odot}$ models of WHW02. As Fig. 8 demonstrates, the radiation is "hotter" in the direction of the blasts. It is also "brighter" in those directions. Hence, we suggest that asymmetric neutrino recoil, integrated over a long time after explosion, can contribute significantly and naturally to the final pulsar kick.

In fact, wind/explosion recoil and the neutrino emission anisotropy after explosion commences provide natural mechanisms for imparting kicks to the residual neutron stars. The anisotropic/top-bottom explosion acts like rocket exhaust and momentum conservation does the rest. The magnitude of the effect can be approximated as follows: the recoil force is equal to $\Phi v \dot{M}_{e}$, where $\Phi$ is the average "anisotropy parameter," $v$ is the characteristic wind velocity, and $\dot{M}_{e}$ is the wind mass loss rate. The power poured into the supernova "nebula" by the wind is $1 / 2 \dot{M}_{e} v^{2}$. Integrating both these quantities over time gives the net impulse and explosion energy $(\eta)$, respectively. The impulse is equal to the residue mass $\left(M_{p n}\right)$ times the kick velocity $\left(v_{k}\right)$. Taking the ratio of these two expressions results in a formula for the kick velocity:

$$
v_{k}=2 \eta /\left(M_{p n} v\right) \Phi .
$$

If we assume that the scale of $v$ is set by a sound speed $\left(\sim 30,000-100,000 \mathrm{~km} \mathrm{~s}^{-1}\right)$, we derive that $v_{k} \sim 1000$ $\left(\eta / 10^{51} \mathrm{ergs}\right) \Phi \mathrm{km} \mathrm{s}^{-1}$. The average observed/inferred kick speed is $300-400 \mathrm{~km} \mathrm{~s}^{-1}$ [30,31], so this number is tantalizing. The anisotropy parameter, $\Phi$, can be large, but depends on the stochasticity of the flow. This formula works whether the explosion is driven by neutrinos or sound, and depends only on the wind-like character of the asymmetric explosion and simple momentum conservation.

However, we cannot, at this stage, determine whether the matter recoil or the neutrino recoil will eventually prove the more important. Nevertheless, what has emerged from our simulations is a straightforward mechanism, matter plus neutrino recoils, for imparting a sizable kick to the residue protoneutron star that does not require anything but the breaking of spherical symmetry and the asymmetric explosion that arises naturally without exotic physics.

\section{Accretion-induced collapse: Rapidly-rotating models}

Recently, Dessart et al. [32] performed a study of the AIC of white dwarfs into neutron stars, including the effects of the rapid rotation that must certainly attend accretion by the primary white dwarf on its way to achieving the Chandrasekhar 


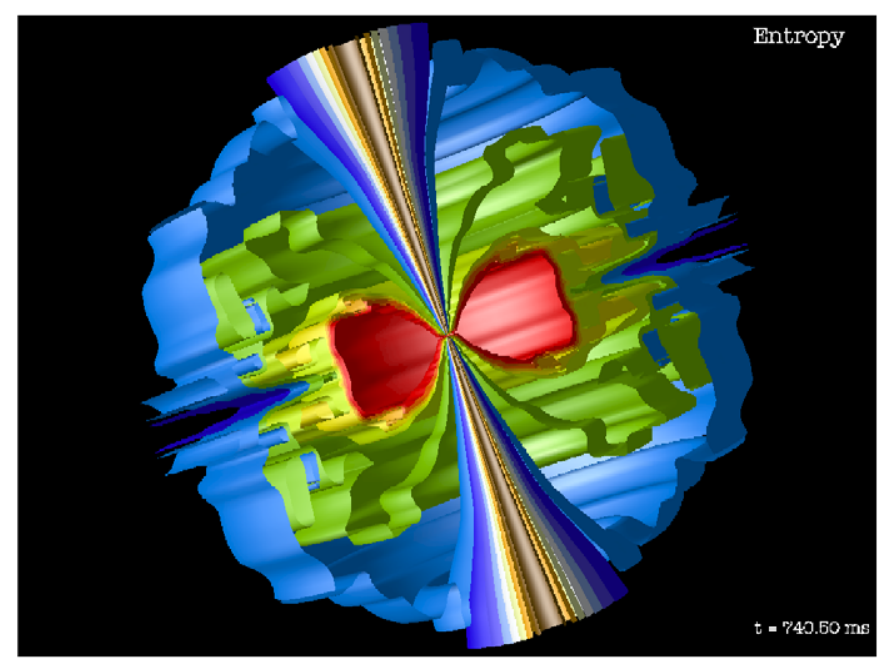

Fig. 9. A 3D still taken during the explosion of the 1.92-M $\odot$ model of Dessart et al. [32] that portrays the bipolar neutrino-driven wind that emerges in this model. The progenitor is a very rapidly rotating white dwarf. Fig. 7 depicts the corresponding degree of angular anisotropy of the neutrinos that emerge from the oblate, spinning protoneutron star. It is this severe anisotropy that translates into the gross asymmetry of the resulting wind.

mass and core collapse. A major distinction between AIC and canonical core collapse is the absence in the former of a significant stellar envelope. Hence, neutrino driving is not inhibited. The shock generated at bounce moves slowly, but steadily, outwards. Within 50-100 ms, the stalled shock breaks out of the white dwarf along the poles. Fig. 9 depicts the bipolar morphology of a representative blast following an AIC. The blast is followed, 200-300 ms after bounce, by a neutrino-driven wind that develops within the excavated white dwarf, in a cone of $\sim 40^{\circ}$ opening angle about the pole, with a mass loss rate of $5-8 \times 10^{-3} \mathrm{M}_{\odot} \mathrm{s}^{-1}$. The ejecta have an entropy on the order of 20-50 $k_{\mathrm{B}} /$ baryon, and the electron fraction is bimodal, with peaks at 0.25 (due to the neutrino-driven wind) and 0.5 (due to the original blast and the wind along the pole). Hence, the AIC of white dwarfs leads to successful explosions with modest energy $\leqslant 10^{50} \mathrm{erg}$, thus comparable to the energies obtained through the collapse of $\mathrm{O} / \mathrm{Ne} / \mathrm{Mg}$ core of stars with $\sim 8-11 \mathrm{M}_{\odot}$ main sequence mass $[13,3]$. This is, however, underenergetic, by a factor of about ten, compared with the inferred value for the core collapse of more massive progenitors leading to Type IIP supernovae. Therefore, the neutrino mechanism can successfully power low-energy explosions of low-mass progenitors and AICs due to the limited mantle mass and steeply declining accretion rate. While the total electron-neutrino luminosities are comparable to those observed in standard core-collapse simulations, the effects of rotation are to reduce the " $v_{\mu}$ " and $\bar{v}_{e}$ luminosities, the latter by one order of magnitude. Additionally, the neutron stars resulting from such AIC of white dwarfs are strongly aspherical, with neutrinospheres having disk-like shapes, akin to apolar-pinched oblate surface. In the faster rotating model $\left(1.92-\mathrm{M}_{\odot}\right)$ of the Dessart et al. [32] study, this configuration results in a strong latitudinal dependence of the neutrino flux, enhanced in the polar direction and reduced in the equatorial direction compared to a non-rotating case (see Fig. 7).

The deleptonized region connected to the neutrinosphere has a butterfly, rather than a spherical shape. Moreover, the neutrino-driven wind originating in the vicinity of the distorted neutrinosphere sees a lower electron-neutrino flux further away from the poles, resulting in a latitudinal dependence of the electron fraction of the ejected material. Fig. 10 depicts the $Y_{e}$ distribution of the ejecta of an AIC explosion, and compares it to the corresponding distribution for our simulation of the 11.2- $\mathrm{M}_{\odot}$ model of WHW02. Superposed are the $2 \mathrm{D} r-z$ velocity vector fields. Compared to standard core collapse, our AIC simulations show no perceptible signs of convection associated with the negative lepton radial-gradient in the protoneutron star, no $\ell=1,2$ oscillations associated with the vortical-acoustic instability, no late time core oscillations, and no sizable neutron star kick. Moreover, in all models, a quasi-Keplerian $0.1-0.5 \mathrm{M}_{\odot}$ disk remains in the equatorial region, that should later be accreted by the neutron star on longer, viscous timescales of many seconds. Fig. 11 is a 3D rendering, with 3D velocity vectors, of an explosive stage of an AIC simulation. The quasi-Keplerian regions and the polar ejecta are clearly indicated with the $3 \mathrm{D}$ velocity vector field. 

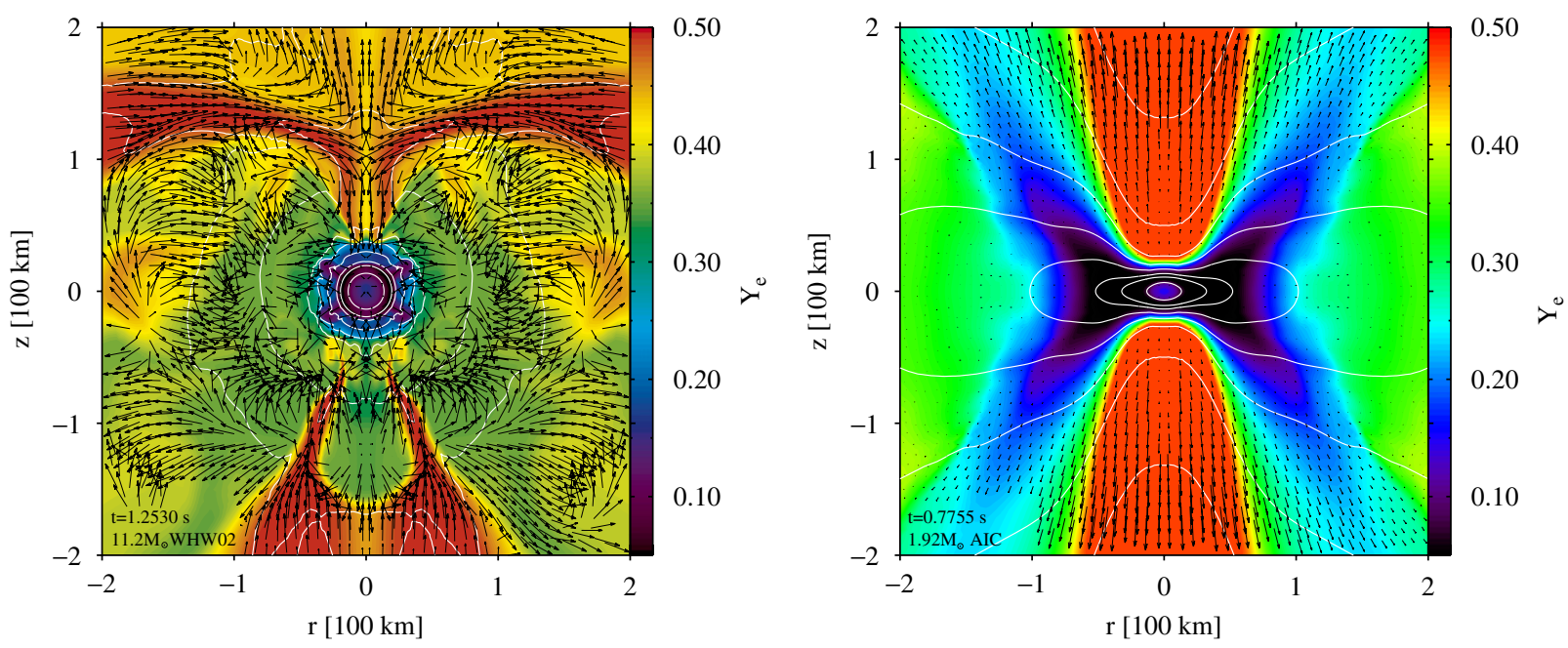

Fig. 10. Colormap of the electron fraction for the 11.2- $\mathrm{M}_{\odot}$ model of WHW02 at $1.253 \mathrm{~s}$ (left) and for the 1.92-M $\odot$ AIC model of Dessart et al. [32] at $0.7755 \mathrm{~s}$ (right), both times since bounce. Velocity vectors are overplotted in black, with a length saturated to $5 \%$ of the width of the display at $5 \times 10^{8} \mathrm{~km} \mathrm{~s}^{-1}$. Note the large convective motions in the left panel, occurring between the shock region and the surface of the protoneutron star. By contrast, the AIC model exhibits a strong neutrino driven wind along the poles, with little or no convection within the protoneutron, in part inhibited by the fast rotation of the corresponding layers.

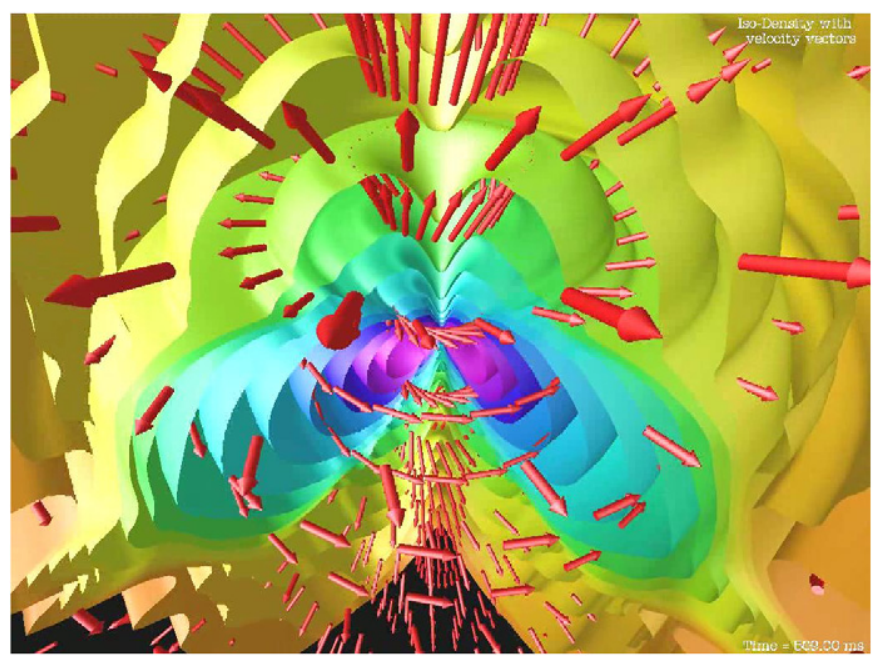

Fig. 11. A snapshot employing isodensity sheets during the explosive phase of the simulation of a rapidly rotating white dwarf created in accretion-induced collapse. The arrows indicate the direction and magnitude of the velocity vectors, including the effect of rotation. This model explodes along the poles, leaving behind a thick disk of material (Simulation performed and published by Dessart et al. [32].)

\section{Core convection effects in the protoneutron star}

Because neutrino heating might still be an important factor in the supernovae mechanism, an enhancement in the neutrino flux during the first second following core bounce could certainly facilitate explosion. Convection in the nascent protoneutron star (PNS) has been invoked as a potential mechanism for such an increase in the neutrino luminosity. Neutrino escape at and above the neutrinosphere establishes a negative lepton-gradient, a situation unstable to convection under the Ledoux criterion. However, Lattimer and Mazurek [33] challenged the idea of large-scale overturn, noting the presence of a positive, stabilizing entropy gradient—a residue of the shock birth. 
In addition, Mayle and Wilson [5-7] have argued that regions stable to convection according to the Ledoux criterion could be sites of doubly-diffusive instabilities, taking the form of so-called neutron (low- $Y_{e}$ material) fingers. This idea rests essentially on the assumption that the neutrino-mediated diffusion of heat occurs on shorter timescales than the neutrino-mediated diffusion of leptons. By contrast, Bruenn and Dineva [34] and Bruenn et al. [35] demonstrated that the neutrino-mediated thermal-diffusion timescale is longer than that of neutrino-mediated lepton-diffusion, and, thus, that neutron fingers do not obtain. Because $v_{\mu}$ 's and $v_{\tau}$ 's have a weak thermal coupling to the material, Bruenn et al. [35] concluded that lepton-diffusion would occur faster by means of low-energy $\bar{v}_{\mathrm{e}}$ 's and $v_{\mathrm{e}}$ 's. Applying their transport simulations to snapshots of realistic core-collapse simulations, they identified the potential for two new types of instabilities within the PNS, referred to as "lepto-entropy fingers" and "lepto-entropy semiconvection."

To study these issues, Dessart et al. [36] have recently performed VULCAN/2D simulations of core convection in the protoneutron star all the way to the center and including lateral neutrino transport. Our conclusions were threefold: First, we do not see any large-scale overturn of the inner PNS material. Second, we see no evidence of doubly-diffusive instabilities in the PNS, expected to operate on diffusion timescales of at least a second, but instead observe the presence of convection, within a radius range of $10-20 \mathrm{~km}$, operating with a timescale of a few milliseconds. Indeed, convection, directly connected to the PNS, is found to occur in two distinct regions: between 10 and $20 \mathrm{~km}$, coincident with the region of negative lepton gradient, and exterior to the PNS above $50 \mathrm{~km}$. However, these two regions are separated by an interface, which shows no sizable outward or inward motion and efficiently shelters the inner PNS.

We find that such convective motions do not appreciably enhance the $v_{e}$ neutrino luminosity, and that they can enhance the $\bar{v}_{e}$ and " $v_{\mu}$ " luminosities by no more than $\sim 15 \%$ and $\sim 30 \%$, respectively, during the first post-bounce $\sim 100 \mathrm{~ms}$, after which the optical depth barrier between the inner convection and the neutrinospheres effectively isolates one from the other, terminating even this modest enhancement. PNS convection is thus found to be a secondary feature of the core-collapse phenomenon, rather than a decisive ingredient for a successful explosion. Furthermore, the typical timescale associated with such convective transport is of the order of a few milliseconds, and thus is at least a thousand times faster than typical growth rates for instabilities associated with neutrino-mediated thermal and lepton diffusion. Such doubly-diffusive instabilities are, therefore, unlikely to play a substantial role in the early critical phases of the PNS. Hence, we concluded that inner PNS motions do not bear importantly on the potential success of core-collapse supernovae explosions.

\section{Gravitational radiation from supernovae}

In the past, model calculations estimating supernova signatures have focussed on the gravitational-wave emission from rotating iron core collapse and core bounce [37]. Recent results from stellar evolutionary calculations [38-40] and neutron star birth spin estimates [41] indicate that presupernova stellar iron cores may rotate much more slowly than previously assumed and that the asphericity during collapse and bounce due to rotation is not generally great enough to produce a sizable time-varying, wave-emitting mass-quadrupole moment. In fact, gravitational radiation from large-scale post-bounce convection and anisotropic neutrino emission are likely to exceed the bounce signal of such slowly rotating supernova cores.

The first-generation LIGO-class detectors are now operating at design sensitivity and an international network of observatories, including LIGO, GEO600, VIRGO and TAMA, is on-line. Gravitational waves detected from a supernova can provide us with "live" dynamical information from the supernova core, complementing the supernova neutrino pulse as the only other immediate carriers of information from deep inside the star. Using signal-processing techniques operating on a large set of theoretical templates, it will be possible to extract supernova physics from a sufficiently strong signal.

The core-oscillation/acoustic mechanism introduces the intriguing possibility that strong gravitational waves can arise from its quadrupole spatial components and, as a result, Ott et al. [42] obtain new estimates for the gravitationalwave signature of core-collapse supernovae. When the $\ell=1$ g-mode grows strong, it starts transferring energy through nonlinear effects to the harmonic at twice its frequency. This harmonic has some $\ell=2$ spatial character. In addition, in some of our models, particularly the more massive ones, fundamental $\ell=2 \mathrm{~g}$-modes themselves are excited. As a result, Ott et al. [42] found that the gravitational waves from the quadrupole components of the core oscillations, if they occur, can dominate the total gravitational wave signature in duration, maximum strain, and total energy emission by one to several orders of magnitude. They have also discovered an approximate progenitor dependence: more massive iron cores may experience higher frequency, higher amplitude oscillations, and, hence, more energetic gravitational-wave 
emission. Note that the acoustic model predicts a delay between core bounce and the onset of the significant phase of gravitational wave emission. It also suggests that during this phase the frequency spectrum of the waves is much more narrow than in previous theories. These are signatures of and tests for the core oscillation model.

\section{Numerical issues}

For these simulations, we have used the two-dimensional multi-group, multi-neutrino-species, flux-limited diffusion (MGFLD) variant of the code VULCAN/2D [22,23,32,36,41-44]. This is currently the only extant 2D, multi-group code that allows core translational motion by introducing a Cartesian-like grid in the inner core and, hence, that is capable of investigating the core-oscillation/acoustic mechanism. Fig. 12 gives an example of such a grid. Note the transition "nodes" at the juncture between the inner and outer griddings. VULCAN/2D is still the only extant supernova code to perform 2D (not "ray-by-ray") multi-group transport with general gravity all the way to the center. Due to the finite-difference character of 2D codes that employ spherical coordinates all the way to the center, to the singularity in those coordinates at that center, and to the reflecting boundary condition frequently imposed at this center, sphericalcoordinate codes are likely to inhibit core translational motions artificially and, hence, to inhibit the $\ell=1 \mathrm{~g}$-modes that are central to the mechanism we have identified. Be that as it may, there are many caveats to our study the reader should keep in mind: (1) Our calculations are Newtonian and not general-relativistic; (2) We employ an approximate (MGFLD, not multi-angle) multi-group transport algorithm in the neutrino sector; (3) Numerical errors are bound to have accumulated due to the need to calculate for $\sim 1,000,000$ timesteps for each progenitor; (4) The initial seed perturbations are unknown (and unknowable?); (5) We are performing the calculations in 2D, not 3D; (6) The Dopplershift terms in the transport equation have been dropped. While these velocity-dependent terms are very different in the laboratory-frame formulation [45] we have adopted than in the comoving frame formulation of Buras et al. [2], they should nevertheless be incorporated; (7) We have used only 16 energy groups; using more $(\geqslant 20)$ is preferred [46]; (8) The transition in the grid from Cartesian-like to spherical coordinates introduces slight artifacts in the flow at the associated topological junctures. The spatial resolution in the center is good, but can be improved on the outside exterior to $\sim 200 \mathrm{~km}$; and (9) The opacities employed are sophisticated [47], but the neutrino-matter correlation effects at higher densities need a second look.

Importantly, the flow is fundamentally chaotic and a precise mapping between initial configuration and final outcome is not possible. This multi-dimensional radiation/hydrodynamical problem is quintessentially meteorological in character. Nevertheless, ours are the first calculations to explore the novel core oscillation/acoustic mechanism and to venture into the late-time behavior of multi-D core collapse with multi-D core motions and multi-D/multi-group transport. What the actual and relative contributions of sound and neutrinos are to the supernova phenomenon as a function of progenitor remains to be determined and will require even more sophisticated numerical tools than we have applied to date to reach a definitive answer. It may be that the neutrino mechanism still obtains in the generic case and that the resulting earlier explosion aborts the acoustic mechanism.

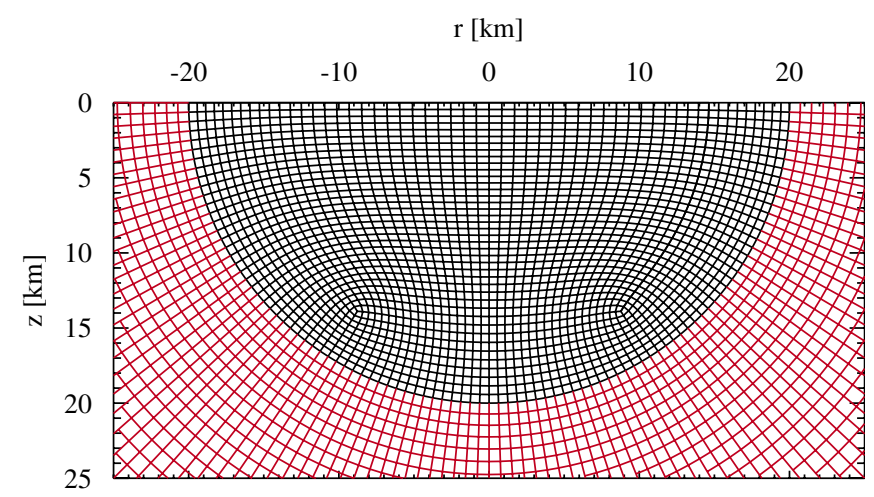

Fig. 12. Illustration of the VULCAN/2D unstructured grid in the central region where it transitions from spherical-polar (red) to Cartesian (black). The spherical-polar region extends from the transition radius at $20 \mathrm{~km}$ out to a maximum radius, with at least 141 logarithmically spaced points. A relaxation method is used to construct the node positions in the inner region, starting with 40 equally spaced points. 


\section{BETHE: a new multi-dimensional radiation hydrodynamic code}

Many phenomena in the Universe must be addressed using the tools of radiation transport, radiative transfer, and radiation hydrodynamics to achieve a theoretical understanding of their character. As this monograph demonstrates, supernovae are among them and for them neutrinos are the radiation. However, whether the radiation is neutrinos or photons, time-dependent techniques to address radiation transport and the coupling of radiation with matter are of central concern to the theorist whose goal is explaining astrophysical phenomena. Moreover, spherically-symmetric algorithms are often not sufficient and multi-dimensional approaches are called for. These are not easy, not only to formulate, but to implement. Nevertheless, multi-D radiation transport is emerging as a necessary tool in the theorist's toolbox and computers to evolve the associated equations are becoming available to a wider cohort of researchers.

In that spirit, Hubeny and Burrows [45] have derived and are starting to implement a new mixed-frame, multi-D, velocity-dependent radiation code that is a departure from the approaches employed to date in supernova theory and in astrophysics. The mixed-frame approach, in which the radiation quantities are defined in the laboratory (Eulerian) frame and the matter and coupling quantities are defined in the comoving frame, has largely been neglected by the radiation transport and atmospheres communities because of their focus on line transfer. Their need to include narrow spectral lines and to handle Doppler shifts into and out of those lines necessitates many spectral bins and a large number of angular bins to ensure the lines are resolved on the computational grid. As a result, most dynamic atmosphere and radiation studies are done using the comoving (Lagrangian) equations of radiation transport. However, since many radiation-hydrodynamic problems do not require an exquisite treatment of spectral line transport, but a good treatment of continuum transport, the mixed-frame methodology is perfectly well suited to many astrophysical problems. Its virtues vis à vis the comoving-frame approach to the solution of the Boltzmann equation and its related moment equations are numerous: (1) Even in one-dimension, instead of requiring $\sim 20$ velocity-dependent terms on the lefthand(operator) side of the Boltzmann/transport equation, many of which involve spatial velocity derivatives, there are no such terms on the left-hand-side in the mixed frame approach and only one grouped linear term (to $O(v / c)$ ) on the right-hand(source) side; (2) There are no terms with derivatives of the velocity. Therefore, the characteristics of the associated transport equation are all straight lines. Furthermore, there is no need for the monotonicity in the velocity field required by some implicit solvers; and (3) The mixed-frame method is easily generalized to two and three dimensions, and the associated solvers are straightforward (though more expensive) extensions of those employed in 1D. Note that much has been made of the importance of velocity-dependent terms in the transport equations for the calculation of the neutrino energy deposited in the "net-gain" [48] region. However, the mixed-frame approach provides the clearest perspective from which to understand the physics of this effect and to see it as simple Doppler shifts of the intensity and neutrino energies. The comoving frame approach obscures this straightforward fact.

We have christened our new radiation-hydrodynamics code employing the mixed-frame philosophy BETHE. ${ }^{1}$ Our formulation is multi-group and multi-angle and includes anisotropic scattering, frequency(energy)-dependent scattering and absorption complete velocity dependence to order $v / c$, rotation, and energy redistribution due to inelastic scattering. Hence, the " $2 \mathrm{D}$ " realization is actually "6 $1 / 2$ "-dimensional. The effects of radiation viscosity are automatically incorporated and we have developed accelerated-lambda-iteration, Krylov subspace (GMRES), discontinuous-finiteelement, and Feautrier numerical methods for solving the equations. Such new algorithms are necessary to improve sufficiently upon what has been done before and explore the viability of both the neutrino and the acoustic mechanisms. Despite the new ideas summarized in this paper, we would not claim that the supernova problem is close to being solved. Far from it. There are always numerical issues that cloud the interpretations of numerical results and constant evolution of technique is necessary to help validate or refute theory. However, we hope that the new code, BETHE, will prove to be a step forward in the ongoing quest to understand one of Nature's most vexing theoretical puzzles and that Hans, who did so much to advance and promote supernova research, would have looked upon our all-too-immodest and inadequate gesture to honor him with an approving smile.

\section{Acknowledgements}

We acknowledge partial support for this work from the Scientific Discovery through Advanced Computing (SciDAC) program of the DOE, Grant no DE-FC02-01ER41184, and from the NSF under Grant AST-0504947.

\footnotetext{
${ }^{1}$ Basic (two-dimensional), Explicit/implicit, transport and hydrodynamics explosion (Code).
} 


\section{References}

[1] H. Bethe, J.R. Wilson, Astrophys. J. 295 (1985) 14.

[2] R. Buras, M. Rampp, H.-Th. Janka, K. Kifonidis, Astron. Astrophys. 447 (2006) 1049.

[3] R. Buras, H.-Th. Janka, M. Rampp, K. Kifonidis, Astron. Astrophys. 457 (2006) 281.

[4] M. Liebendörfer, A. Mezzacappa, F.-K. Thielemann, O.E.B. Messer, W.R. Hix, S.W. Bruenn, Phys. Rev. D 63 (2001) 103004.

[5] J.R. Wilson, R. Mayle, Phys. Rep. 163 (1988) 63.

[6] J.R. Wilson, R. Mayle, Phys. Rep. 227 (1993) 97.

[7] R. Mayle, J.R. Wilson, Astrophys. J. 334 (1988) 909.

[8] M. Herant, W. Benz, W.R. Hix, C.L. Fryer, S.A. Colgate, Astrophys. J. 435 (1994) 339.

[9] A. Burrows, J. Hayes, B.A. Fryxell, Astrophys. J. 450 (1995) 830.

[10] H.-Th. Janka, E. Müller, Astron. Astrophys. 306 (1996) 167.

[11] C.L. Fryer, M. Warren, Astrophys. J. 574 (2002) L65.

[12] C.L. Fryer, M. Warren, Astrophys. J. 601 (2004) 391.

[13] F.S. Kitaura, H.-Th. Janka, W. Hillebrandt, Astron. Astrophys. 450 (2006) 345.

[14] K. Nomoto, M. Hashimoto, Phys. Repts. 163 (1988) 13.

[15] S.E. Woosley, A. Heger, T.A. Weaver, Rev. Mod. Phys. 74 (2002) 1015.

[16] J.M. Blondin, A. Mezzacappa, C. DeMarino, Astrophys. J. 584 (2003) 971.

[17] J.M. Blondin, A. Mezzacappa, Astrophys. J. 642 (2006) 401.

[18] T. Foglizzo, M. Tagger, Astron. Astrophys. 363 (2000) 174.

[19] T. Foglizzo, Astron. Astrophys. 368 (2001) 311.

[20] T. Foglizzo, Astron. Astrophys. 392 (2001) 353.

[21] T. Foglizzo, P. Galletti, M. Ruffert, Astron. Astrophys. 435 (2005) 397.

[22] A. Burrows, E. Livne, L. Dessart, C.D. Ott, J. Murphy, Astrophys. J. 640 (2006) 878.

[23] A. Burrows, E. Livne, L. Dessart, C.D. Ott, J. Murphy, Astrophys. J. 655 (2007) 416.

[24] T. Foglizzo, P. Galletti, L. Scheck, H.-Th. Janka, Astrophys. J. 654 (2006) 1006.

[25] A. Burrows, J. Goshy, Astrophys. J. 416 (1993) 75.

[26] L. Scheck, T. Plewa, H.-Th. Janka, K. Kifonidis, E. Müller, Phys. Rev. Lett. 92 (2004) 011103.

[27] L. Scheck, K. Kifonidis, H.-Th. Janka, E. Müller, Astron. Astrophys. 457 (2006) 963.

[28] L. Wang, D. Baade, P. Höflich, J.C. Wheeler, Astrophys. J. 592 (2003) 457.

[29] D.C. Leonard, et al., Nature 440 (2006) 505.

[30] J.H. Taylor, J.M. Cordes, Astrophys. J. 411 (1993) 674.

[31] A. Lyne, D.R. Lorimer, Nature 369 (1994) 127.

[32] L. Dessart, A. Burrows, C.D. Ott, E. Livne, S.-Y. Yoon, N. Langer, Astrophys. J. 644 (2006) 1063.

[33] J.M. Lattimer, T.J. Mazurek, Astrophys. J. 246 (1981) 955.

[34] S.W. Bruenn, T. Dineva, Astrophys. J. 458 (1996) L71.

[35] S.W. Bruenn, E.A. Raley, A. Mezzacappa, astro-ph/0404099, 2005.

[36] L. Dessart, A. Burrows, E. Livne, C.D. Ott, Astrophys. J. 645 (2006) 534.

[37] C.D. Ott, A. Burrows, E. Livne, R. Walder, Astrophys. J. 600 (2004) 834.

[38] A. Heger, S.E. Woosley, H. Spruit, Astrophys. J. 626 (2005) 350.

[39] R. Hirschi, G. Meynet, A. Maeder, Astron. Astrophys. 425 (2004) 649.

[40] R. Hirschi, G. Meynet, A. Maeder, Nucl. Phys. A 758 (2005) 234.

[41] C.D. Ott, A. Burrows, L. Dessart, E. Livne, Astrophys. J. (Suppl.) 164 (2006) 130.

[42] C.D. Ott, A. Burrows, L. Dessart, E. Livne, Phys. Rev. Lett. 96 (2006) 201102.

[43] E. Livne, A. Burrows, R. Walder, T.A. Thompson, I. Lichtenstadt, Astrophys. J. 609 (2004) 277.

[44] R. Walder, A. Burrows, C.D. Ott, E. Livne, I. Lichtenstadt, M. Jarrah, Astrophys. J. 626 (2005) 317.

[45] I. Hubeny, A. Burrows, Astrophys. J., 2006, submitted for publication.

[46] T.A. Thompson, A. Burrows, P.A. Pinto, Astrophys. J. 592 (2003) 434.

[47] A. Burrows, T.A. Thompson, Neutrino-matter interaction rates in supernovae: The essential microphysics of core collapse, in: C. Fryer (Ed.), Core Collapse of Massive Stars, Kluwer Academic Press, Dordrecht, 2004, p. 133.

[48] J.R. Wilson, in: J. Centrella, J.M. LeBlanc, R.L. Bowers (Eds.), Numerical Astrophysics, Jones and Bartlett, Boston, 1985 , p. 422. 